\title{
A methodology to determine the elastic moduli of crystals by matching experimental and simulated lattice strain pole figures using discrete harmonics
}

\author{
Euan Wielewski ${ }^{1}$, Donald E. Boyce ${ }^{2}$, Jun-Sang Park ${ }^{3}$, Matthew P. \\ Miller $^{2}$ and Paul R. Dawson ${ }^{2}$ \\ ${ }^{1}$ School of Engineering, University of Glasgow, UK \\ ${ }^{2}$ Sibley School of Mechanical and Aerospace Engineering, Cornell University, USA \\ ${ }^{3}$ Advanced Photon Source, Argonne National Laboratory, USA
}

November 14, 2016

\begin{abstract}
Determining reliable single crystal material parameters for complex polycrystalline materials is a significant challenge for the materials community. In this work, a novel methodology for determining those parameters is outlined and successfully applied to the titanium alloy, Ti-6Al-4V. Utilizing the results from a lattice strain pole figure experiment conducted at the Cornell High Energy Synchrotron Source, an iterative approach is used to optimize the single crystal elastic moduli by comparing experimental and simulated lattice strain pole figures at discrete load steps during a uniaxial tensile test. Due to the large number of unique measurements taken during the experiments, comparisons were made by using the discrete spherical harmonic modes of both the experimental and simulated lattice strain pole figures, allowing the complete pole figures to be used to determine the single crystal elastic moduli.
\end{abstract}

\section{Introduction}

Microstructurally detailed, crystal-scale simulations are increasingly being used to better understand and predict the mechanical behavior of engineering materials. The predictive capabilities of these crystal-scale simulations are fundamentally dependent on the single crystal material properties (such as

(C) 2016. This manuscript version is made available under the Elsevier user license http://www.elsevier.com/open-access/userlicense/1.0/ 
elastic moduli and critical resolved shear stresses) used for the constituent phases.

For the elastic response, the moduli appear as components of the elasticity tensor, $\mathcal{C}(\boldsymbol{r})$, in Hooke's law, written here using the unstressed configuration as a reference volume:

$$
\boldsymbol{\tau}=\mathcal{C}(\boldsymbol{r}) e^{e}
$$

where $\boldsymbol{\tau}$ is the Kirchhoff stress and $e^{e}$ is the elastic strain. The anisotropic behavior stemming from the crystal symmetry arises through the orientation dependence of $\mathcal{C}(\boldsymbol{r})$, which is indicated by its argument, $\boldsymbol{r}$, the Rodrigues vector for the lattice orientation. An accurate simulation of a polycrystalline aggregate requires high fidelity moduli of the constituent phases.

Traditionally, determination of the single crystal elastic stiffness tensor involves constructing mechanical tests on a single crystal specimen that systematically isolates each term [1]. For nearly a century, quasi static [2-4], and dynamic loading experiments - including ultrasonic probes [5-9] - have been conducted on single crystals to quantify elastic moduli. X-rays have also been used to quantify lattice strains within single crystal specimens subjected to simple, often uniaxial, loading conditions [10, 11].

However, manufacturing single crystal specimens with the same chemistry as engineering alloys can be problematic. As such, methods are required that are capable of determining the elastic moduli of the constituent phases from polycrystalline specimens. If the microstructure within a polyphase metal can be approximated with a simple morphology - such as a lattice ultrasonic methods can be used to approximate the moduli of each phase [12]. Lattice strains from individual grains within a loaded aggregate can also be measured using high energy synchrotron x-ray diffraction [13]. Individual grain strains can be used to estimate the elastic moduli in conjunction with models for polycrystal responses.

For example, along with a crystal-based finite element simulation, the lattice strains from several individual crystals within a deforming aggregate were used to quantify the single crystal moduli of a BCC titanium alloy [14]. Often, due to the small grain sizes present in engineering alloys, one cannot obtain diffraction data from individual crystals and experiments that measure average strains over an aggregate is the only option. In the past, the diffraction elastic constants - often employed in the determination of residual stress - and several lattice strains measured under uniaxial loading conditions have been inverted for components of the single crystal moduli tensor using both lab source x-rays [15-17] and neutrons $[18,19]$. A possible disadvantage 
of these methods is the assumption that each crystal is subjected to a uniaxial stress state. In situ strain pole figure experiments employing a uniaxial test specimen [20] and associated finite element simulations have shown that the stress state can vary significantly over a polycrystalline sample [21].

In this paper, we present a methodology for determination of the single crystal elastic moduli of the hexagonal close-packed (HCP) $\alpha$ phase of a Ti$6 \mathrm{Al}-4 \mathrm{~V}$ specimen subjected to uniaxial tension. The lattice strains in the $\alpha$ phase were measured during mechanical loading using in situ high energy x-ray diffraction measurements. The challenge is to accurately represent the stress state of the crystals participating in each diffraction measurement, then to recover the moduli by comparing the simulations to the experiment. We employ a crystal-scale finite element model to simulate the experiment and a discrete spherical harmonic-based data analysis technique to compare the simulated and experimental lattice strain pole figures. The discrete spherical harmonic analysis allows the lattice strain trends over the entire polycrystal to be extracted and for noise in the data to be isolated. An optimization routine has been implemented to determine the single crystal elastic moduli that give the best fit between the experimentally measured and simulated lattice strain distributions.

\section{Moduli Optimization Methodology}

The changes in lattice strain that occur with increments of load in the elastic regime can be used to estimate the elastic moduli. The estimation method presented here consists of finding the set of moduli that minimize the differences between simulated and measured lattice strain distributions. The lattice strain distributions are available in the form of lattice strain pole distributions (also referred to as strain pole figures, SPF). In the limit of infinite resolution, a strain pole distribution has infinite degrees of freedom, but in practice is represented approximately with a number of parameters determined by the extent of the data defining it. With currently available experimental methods, this number can be relatively large - on the order of thousands for each SPF.

The methodology presented here limits the degrees of freedom used to represent the measured and simulated strain distributions by framing the minimization in terms of the coefficients of the spherical harmonic expansions used to represent the lattice strain distributions over the unit sphere. In addition to curtailing the size of the optimization problem, the expansions effectively smooth the distributions and draw out the dominant trends. 
In a polycrystal subjected to mechanical loads, the crystals are strained, and because the crystals are elastically and plastically anisotropic, the strain varies spatially over the volume of the polycrystal. The value of the strain observed within a specific grain depends on many factors, including the orientation of the lattice, the relative strengths and stiffnesses of surrounding grains, and, of course, the type and intensity of the loading. Again owing to the anisotropy of the properties, the lattice orientation plays a primary role, such that a distribution of strain as a function of orientation can be identified from either experimental or simulation data. Here, we define $\boldsymbol{\epsilon}(\boldsymbol{r})$ as the average strain in crystals with orientation $\boldsymbol{r}$. It is defined over the fundamental region of orientation space, $\Omega_{\mathrm{fr}}$. See Appendix A for additional explanation of the orientation space parameterization used in this paper.

Crystallographic fibers are loci within the fundamental region on which crystals share a common orientation up to a rotation about one axis [22, 23]. If we designate this axis as a sample direction, $\boldsymbol{s}$, then the condition satisfied by all crystals lying on a common fiber is:

$$
c(\boldsymbol{r})= \pm s
$$

where $\boldsymbol{c}(\boldsymbol{r})$ includes both the designated crystallographic plane for a crystal with orientation, $\boldsymbol{r}$, and its symmetric equivalents. A fiber defined by Equation 2 is designated by $\Upsilon_{c \| s}$.

The normal component of the strain within a crystal with orientation $\boldsymbol{r}$ in the direction parallel to the sample direction, $\boldsymbol{s}$, is $\boldsymbol{s} \cdot \boldsymbol{\epsilon}(\boldsymbol{r}) \cdot \boldsymbol{s}$. The average value of this strain component for all the crystals satisfying Equation 2 is obtained by integrating along the crystallographic fiber, $\Upsilon_{c \| s}$ :

$$
\epsilon^{c \| s}=\int_{\Upsilon_{c \| s}} A(\boldsymbol{r}) \boldsymbol{s} \cdot \boldsymbol{\epsilon}(\boldsymbol{r}) \cdot \boldsymbol{s} \mathrm{d} \Upsilon
$$

where $A(\boldsymbol{r})$ is the texture and the average value of the strain component is designated as $\epsilon^{c \| s}$. As is evident from its definition, $\epsilon^{c \| s}$ depends on both $\boldsymbol{c}$ and $s$. These dependencies are displayed by constructing distributions of $\epsilon^{c \| s}$ over the unit sphere for each family of crystallographic planes, $\boldsymbol{c}$, of interest. Points on the sphere correspond to sample directions, $\boldsymbol{s}$. These distributions are lattice strain pole figures, SPFs.

The SPF distributions can be represented by a continuous, piecewise polynomial representation over the unit sphere:

$$
\epsilon^{c \| s}(s)=\left[N^{s}(s)\right]\left\{\hat{\epsilon}^{c \| s}\right\}
$$


where $\left[N^{s}(s)\right]$ are interpolation functions with $C^{0}$ continuity and $\left\{\hat{\epsilon}^{c \| s}\right\}$ are nodal point values. Both the measured and simulated strain distributions are represented with Equation 4 and the appropriate sets of the nodal point values: $\left\{\hat{\epsilon}^{c \| s}\right\}_{e}$ for experimental data and $\left\{\hat{\epsilon}^{c \| s}\right\}_{s}$ for the simulated distributions of strain. $\left\{\hat{\epsilon}^{c \| s}\right\}_{e}$ is known from experiments and thus is fixed. $\left\{\hat{\epsilon}^{c \| s}\right\}_{s}$, on the other hand, depends on values of the elastic moduli and will be recomputed as the moduli are varied in search of optimal values.

For both the experimental and simulated distributions, we represent $\left\{\hat{\epsilon}^{c \| s}\right\}$ with a series expansion, in this case a set of discrete spherical harmonics:

$$
\left\{\hat{\epsilon}^{c \| s}\right\}=\sum_{k=1}^{n^{w}} w^{k}\left\{\hat{H}^{k}\right\}
$$

The method for determining the basis functions for the harmonic expansion is presented in Appendix B. To evaluate the weights, $w^{k}$, for a given $\left\{\hat{\epsilon}^{c|| s}\right\}$, we first re-write Equation 5 in a matrix form:

$$
\left\{\hat{\epsilon}^{c \mid s}\right\}=[\tilde{H}]\left\{\mathrm{w}^{c}\right\}
$$

where

$$
\left\{\mathrm{w}^{c}\right\}=\left\{\mathrm{w}_{1}^{c} \mathrm{w}_{2}^{c} \mathrm{w}_{3}^{c} \ldots \mathrm{w}_{n^{w}}^{c}\right\}^{\mathrm{T}}
$$

and

$$
[\tilde{H}]=\left[\left\{\hat{H}^{1}\right\}\left\{\hat{H}^{2}\right\}\left\{\hat{H}^{3}\right\} \ldots\left\{\hat{H}^{n^{w}}\right\}\right]
$$

Here, the superscript $c$ refers to a particular reflection for which we have experimental data. To solve for $\left\{w^{c}\right\}$ from Equation 6, we construct the corresponding normal equations using least squares to obtain:

$$
[\tilde{H}]^{\mathrm{T}}[\tilde{H}]\left\{\mathrm{w}^{c}\right\}=[\tilde{H}]^{\mathrm{T}}\left\{\hat{\epsilon}^{c} \| s\right\}
$$

Given $\left\{w^{c}\right\}$ for both the experimental and simulated distributions (designated as $\left\{\mathrm{w}^{c}\right\}_{e}$ and $\left\{\mathrm{w}^{c}\right\}_{s}$, respectively), an error, $R_{w}$, is defined:

$$
R_{w}=\sum_{c}\left[\left\{\mathrm{w}^{c}\right\}_{e}-\left\{\mathrm{w}^{c}\right\}_{s}\right]^{2}
$$

Equation 10 serves as the objective function in the optimization procedure for estimating values of the elastic moduli by minimizing $R_{w}$. The optimization search begins with an initial estimate of the independent moduli, typically taken from handbook references, and iterates on their values to minimize the objective function. The contributions to $R_{w}$ span strain pole figures for all of the crystal plane families at each of the load steps with 
measurements. Iterates for $\left\{w^{c}\right\}_{s}$ are expensive, as they involve performing a finite element simulation as outlined in Section 3.3 using different combinations of the moduli. Consequently, to minimize $R_{w}$, a manual, univariate descent procedure is invoked in which the values of the moduli are varied one at a time to generate the necessary gradient evaluations. The descent process is continued to achieve smallest error and thereby to estimate the set of elastic moduli that provides the best fit overall between the measured and computed lattice strains.

The described methodology is based on the availability of SPF distributions. SPF distributions are directly available from in situ powder diffraction experiments as a consequence of Bragg's Law and rotation of the specimen to populate the pole figure. SPF distributions can also be generated from simulation results by evaluating which crystals in a virtual polycrystal satisfy the Bragg condition for x-rays with a given input vector. Generation of experimental and simulated SPFs are outlined in the following section, as well as a description of the material the methodology has been applied to, the titanium alloy, Ti-6Al-4V.

\section{Material and Lattice Strain Pole Figures}

To demonstrate the methodology for determining single crystal elastic moduli for an engineering alloy, we examine the titanium alloy, Ti-6Al-4V. In this section, the material microstructure is quantified and the lattice strain data are presented.

\subsection{Material}

Ti-6Al-4V is a dual-phase titanium alloy consisting of a HCP $\alpha$ phase and a body-centred cubic (BCC) $\beta$ phase. The $\alpha$ phase typically constitutes $92 \%$ of Ti-6Al-4V by volume and, as such, dominates the elastic response of the material. The material used in this study had a nominal yield stress and Young's modulus of $800 \mathrm{MPa}$ and $110 \mathrm{GPa}$, respectively.

Ti-6Al-4V can be thermo-mechanically processed to have a number of different microstructural forms, ranging from fine transformed lath structures to equiaxed grains. The material used in this study came from the "mill annealed" plate processing route and consisted of equiaxed $\alpha$ grains with an average grain size of $12 \mu \mathrm{m}$ in a matrix of prior- $\beta$. A backscatter electron micrograph of the material is given in Figure 1.

For a particular diffraction volume, the texture intensity pole figures were computed using the Materials Analysis Using Diffraction (MAUD) software 


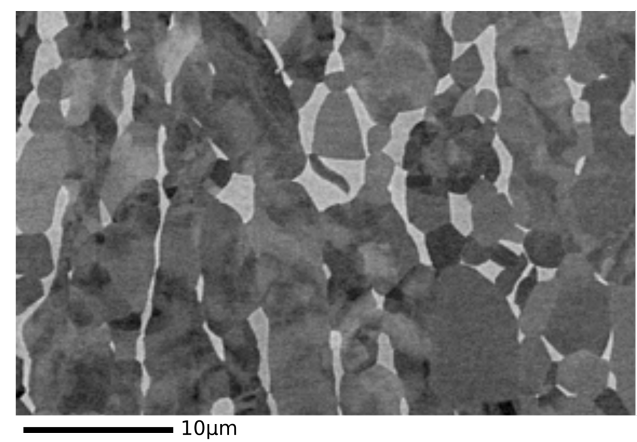

Figure 1: Backscatter electron image showing the microstruture of the millannealed Ti-6Al-4V material. Dark regions are $\alpha$ phase and light regions are $\beta$ phase.

[24]. These intensity pole figures were then used to compute the orientation distribution function (ODF). The OdfPf package ${ }^{1}$ was employed for the ODF calculation. The ODF for the $\alpha$ phase of the Ti-6Al-4V is plotted over the fundamental region of the orientation space for hexagonal crystal symmetry using Rodrigues parameterization in Figure 2, relative to the rolling (RD), transverse (TD) and normal (ND) directions of the plate. This ODF was used to instantiate a virtual polycrystal for the simulation work.

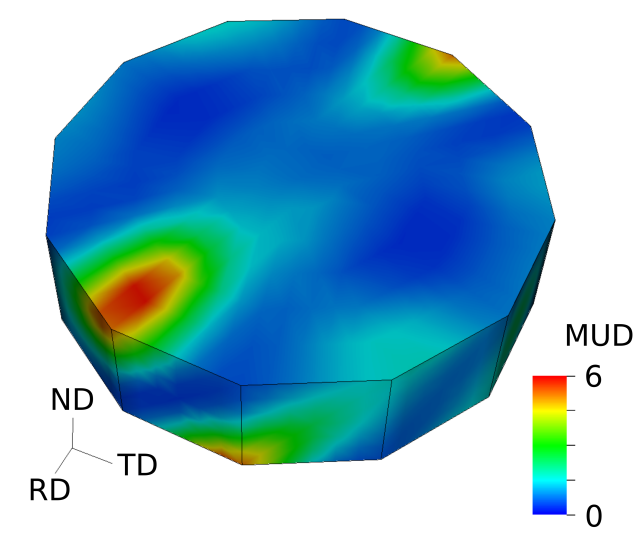

Figure 2: The ODF for the $\alpha$ phase of the Ti-6Al-4V sample. The units are in multiples of uniform distribution (MUD).

\footnotetext{
${ }^{1}$ http://anisotropy.mae.cornell .edu
} 


\subsection{In situ HEXD experiments and measured lattice strain data}

High energy x-ray diffraction (HEXD) measurements were conducted on a Ti-6Al-4V tensile specimen under in situ loading in the A2 experimental station at the Cornell High Energy Synchrotron Source (CHESS). The tensile loads were applied to the specimen using a custom-built, displacementcontrolled load frame. The applied force was measured using a $10 \mathrm{kN}$ load cell and the strain in the gage section of the specimen was measured using a strain gage attached to the surface of the specimen. The tensile specimen had a gage section with a square cross-section of width, $1.8 \mathrm{~mm}$ and length, $8 \mathrm{~mm}$. The loading axis of the tensile specimen was orientated in the rolling direction $(\mathrm{RD})$ of the plate and the square cross-section of the gage section was aligned with the orthogonal transverse (TD) and plate normal (ND) directions. The engineering stress-strain response is given in Figure 3.

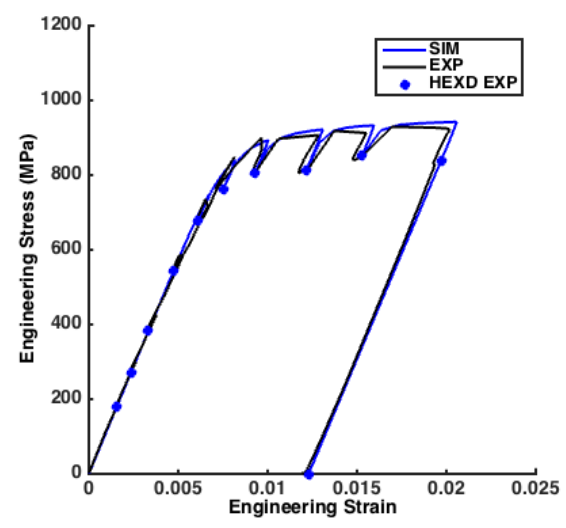

Figure 3: Experimental and simulated engineering stress-strain curves, with HEXD measurement points highlighted.

For the HEXD measurements, the transmission geometry described by Miller et al. [20] was employed. Figure 4 illustrates the experimental geometry. The wavelength of the monochromatic x-ray was $0.02086 \mathrm{~nm}$ (60 $\mathrm{keV})$. The nominal beam size was $500 \mu \mathrm{m} \times 500 \mu \mathrm{m}$. Taking the average grain size $(12 \mu \mathrm{m})$ and assuming spherical grains, this beam size results in approximately 500,000 grains contributing to each diffraction image. An amorphous $\mathrm{Si}$ area detector was placed approximately $941 \mathrm{~mm}$ from the sample to record the full Debye-Scherrer rings. A set of $\mathrm{CeO}_{2}$ images were recorded to characterize the experimental setup. 


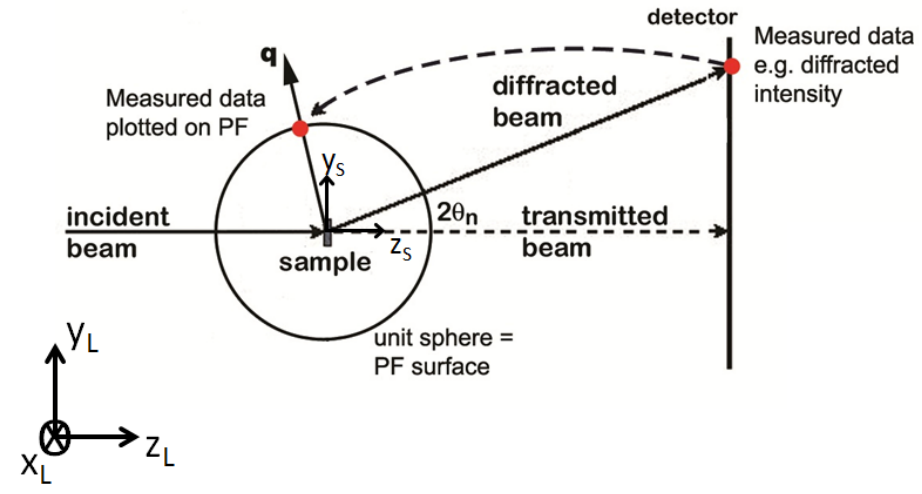

Figure 4: A schematic of the experimental geometry used in this work.

At several points during the loading program, the loading was paused and a set of diffraction images were recorded. To ensure that the specimen did not excessively creep while the diffraction images were recorded, the load was reduced by $10 \%$ from the peak load. At each of these interruptions of the loading, the specimen was rotated with respect to the incident monochromatic x-ray beam by $\pm 75^{\circ}$ about the loading direction, $\mathrm{Y}_{\mathrm{s}}$, to maximize the number of measurement directions while avoiding the shadows from the load frame posts. A schematic of the experimental geometry used is given in Figure 4. During rotation, 15 diffraction images were recorded at equal angular intervals. Furthermore, the specimen was also translated along the loading direction to interrogate six different diffraction volumes in the gage length. In total, ninety diffraction images were recorded at each measurement point of the loading program. Unlike high energy x-ray diffraction microscopy measurements [13], where individual crystals are tracked with changing macroscopic loads, the individual grains within a diffraction volumes were not tracked in this work; here, the underlying assumptions are that:

- the applied load is uniform along the gage length, and

- the volume of material illuminated by the x-ray beam is sufficient to be representative of the material at-large.

For each diffraction volume at a particular macroscopic load, the diffraction images were used to construct the lattice strain pole figures for multiple families of crystallographic planes in the $\alpha$ phase of the Ti-6Al-4V sample. The $\beta$ phase was ignored as its volume fraction was small. For any 
given diffraction volume, the raw strain pole figures were computed using the analysis method outlined in [20]. The lattice strain at particular scattering vector, $\boldsymbol{s}$, for a particular family of crystallographic planes, denoted by $s$, was computed by:

$$
\epsilon^{c \| s}(s)=\frac{d^{c \| s}(s)-d_{o}^{c \| s}(s)}{d_{o}^{c \| s}(s)}
$$

where $d_{o}^{c \| s}(s)$ and $d^{c \| s}(s)$ are the plane spacings of the family of crystallographic planes measured along $s$ while the sample was under load and at zero load, respectively.

The raw lattice SPF data points were used to construct continuous distributions over the unit sphere for three families of crystallographic planes

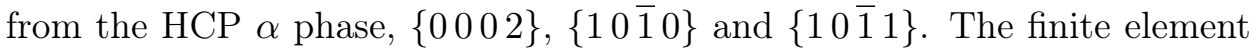
mesh over the unit sphere is shown in Figure 5. The data points were interpolated to the nodal points of the finite element mesh using a biharmonic spline interpolation over the 2-D surface of the unit sphere. With lattice strain values prescribed at every nodal point of the mesh, the lattice strain field is defined completely over the unit sphere via Equation 4. The stages of this process of constructing the continuous SPFs are illustrated in Figure 5.

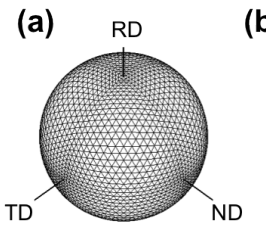

(b)

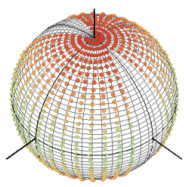

(c)

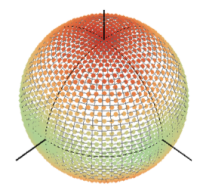

(d)

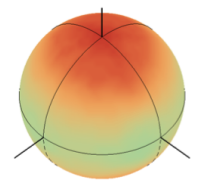

Figure 5: Plots of the unit sphere showing (a) the finite element mesh used to calculate the discrete spherical harmonics, (b) the raw SPF data for an example family of crystallographic planes and loading step, (c) the raw SPF data interpolated to the nodal points of the finite element mesh and (d) the lattice strain field over the unit sphere.

The lattice strain distributions at five load steps within the elastic region

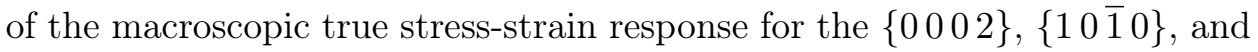
$\{10 \overline{1} 1\}$ crystal plane families are given in Figure 6 for a single diffraction volume. Although the outer rings on the detector provide better strain resolution, the inner three rings where chosen as these gave the most complete coverage over the pole figures. From Figure 6 it can be seen that for all three families of crystallographic planes, the tensile component of the lattice strain 
distribution increases in the RD (loading) direction as the macroscopic load is increased. Further, due to the Poisson effect, a compressive component of the lattice strain distribution develops around the equator of the SPF as the macroscopic load increases.

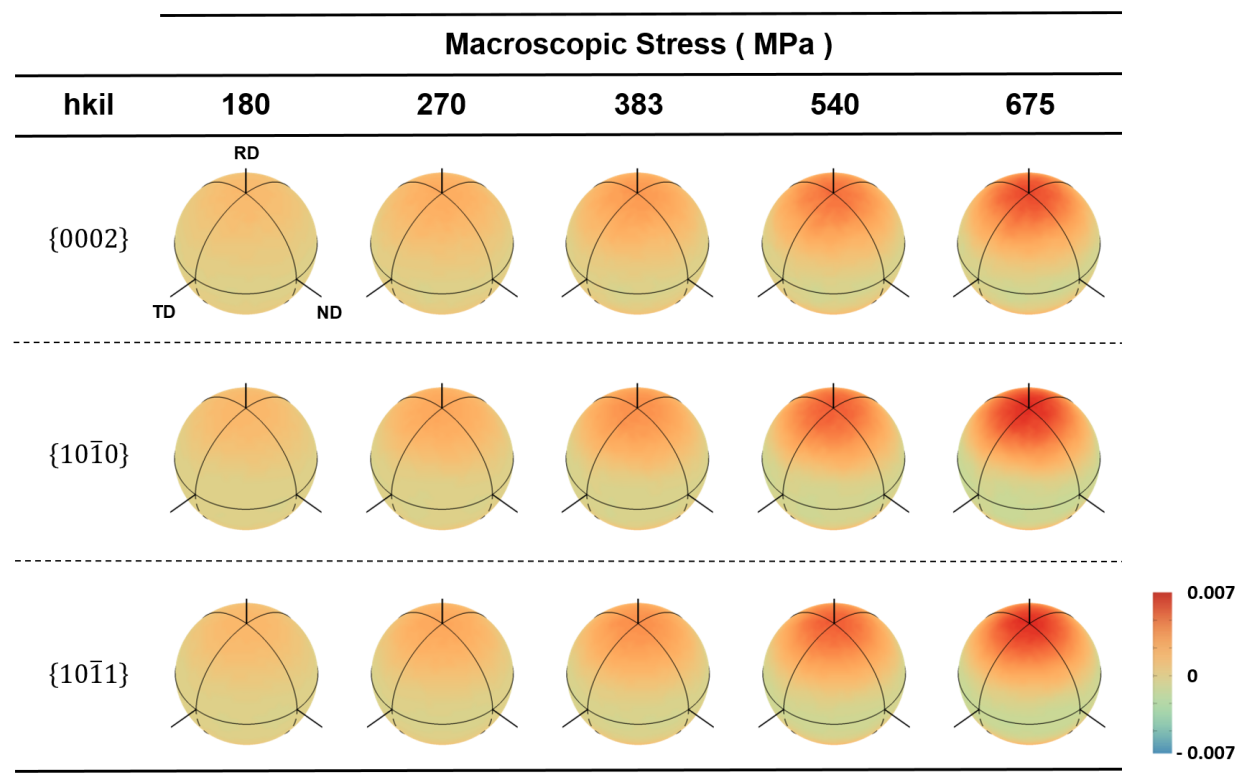

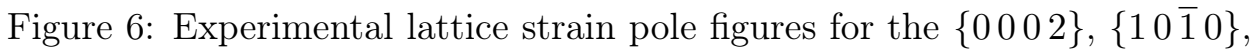
and $\{10 \overline{1} 1\}$ crystal plane families at five macroscopic true stress values in the elastic region of the macroscopic true stress-strain response for a representative volume.

The experimental data rendered a total of $15 \mathrm{SPF}$ distributions within the elastic domain (three crystal plane families, each at five load steps) for six independent diffraction volumes. For each of the $15 \mathrm{SPF}$ conditions, the harmonic expansion coefficients defined in Equations 6 and 7 were evaluated for the six independent diffraction volumes. The error analysis indicated that some sample directions were subject to higher variability in the measured strains than others, a trend that can be traced to the completeness of the experimental coverage [25]. Interestingly, calculating the harmonic expansion coefficients of the standard Error Pole Figure (EPF) (see Appendix C) showed that this variability had a strong influence on the first mode in the harmonic expansion, but tended to have only a weak influence on the higher modes. The first mode is a uniform distribution over the sphere, thus showing no orientation dependence. 


\subsection{Crystal-scale finite element simulations and simulated lattice strain data}

The simulated strain pole figures are computed by simulating the responses of virtual polycrystals using a crystal-scale finite element formulation for the elasto-plastic deformations of polycrystals. Thorough documentation of the finite element formulation together with details of its numerical implementation may be found in [26]. Virtual polycrystals were instantiated with 2000 grains to represent the test specimen. Although the number of grains in the virtual polycrystals are significantly lower than those sampled in the experiment (500,000 grains per diffraction image), 2000 grains was found to be sufficient to accurately capture the observed lattice strain distributions. Each grain is discretized with approximately 50-100 10-node tetrahedral elements. An example of a typical virtual polycrystal used in this study is shown in Figure 7.

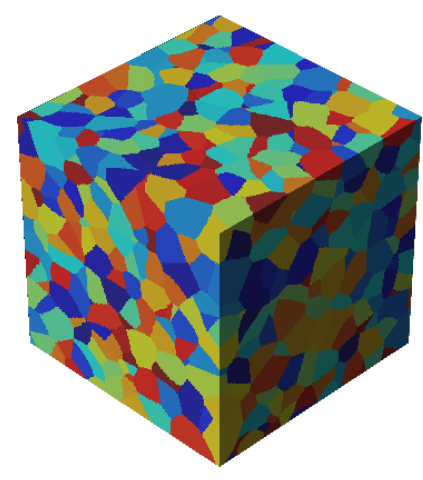

Figure 7: Virtual polycrystal used in HEXD simulations. Color indicates a grain.

Crystal lattice orientations are assigned to the grains by sampling from a known orientation distribution function (ODF) for the material. A baseline set of single crystal elastic moduli is chosen, usually from the literature, to initiate the optimization procedure described in Section 2. The virtual polycrystals are subjected to the same load history as the experiment.

The stress and strain tensors are recorded for every element at every load step. From these records, virtual SPFs are then constructed for each of the load steps and crystallographic reflections of interest. The process of extracting $\epsilon^{c \| s}(s)$ consists of sweeping through all the elements and computing the following quantities: 
1. Given $s$, if $\boldsymbol{c} \| s$, then compute $\epsilon^{c \| s}$

2. Add weighted value to a running total. (Weight = volume element / volume of crystals satisfying: $\boldsymbol{c} \| \boldsymbol{s}$.)

3. Repeat for all $s$ corresponding to nodal points of the mesh on the sphere.

The texture, $A(\boldsymbol{r})$, enters via the instantiation step as orientations were assigned to elements consistent with texture, so weighting of the lattice strains by appropriate volumes is implicit in summing over elements that satisfy the fiber condition.

Figure 3 shows a typical computed stress-strain response on the same plot with the experimental stress-strain results. The simulations included the unloading episodes that were conducted in the experiments to avoid specimen creep during the diffraction measurements. The simulations were carried out for the entire loading history, which extended beyond the yield point. For the evaluation of the elastic moduli the experimental points up to a nominal stress of $675 \mathrm{MPa}$ were considered.

The optimization procedure seeks to find the optimal set of moduli by minimizing the error defined by Equation 10. Each iterate of the procedure involves recomputing the simulated strain pole figures and re-evaluating the expansion coefficients. Thus, the finite element simulations are performed repeatedly with different values of the moduli following a univariant descent procedure. The variations in the moduli were constrained to values that provided a fixed bulk modulus, as explained in Section 4.1.

\section{Optimization of Elastic Moduli}

Using the methodology presented in Section 2 and the lattice strain data presented in Section 3.2, we now evaluate the elastic moduli for the dominant HCP $\alpha$ phase of Ti-6Al-4V. We first examine the stiffness tensor for the HCP phase of Ti-6Al-4V to expose the moduli of interest, discuss constraints on those moduli, and to identify those moduli that bear on the bulk and deviatoric responses. We then discuss the simulations conducted in performing the optimization. This is followed by presentation of the optimized moduli.

\subsection{Elastic moduli for the HCP phase of Ti-6Al-4V}

We re-write Equation 1 in matrix form for the case of hexagonal symmetry to identify the specific moduli of interest: 


$$
\left\{\begin{array}{l}
\tau_{11} \\
\tau_{22} \\
\tau_{33} \\
\tau_{23} \\
\tau_{13} \\
\tau_{12}
\end{array}\right\}=\left[\begin{array}{llllll}
C_{11} & C_{12} & C_{13} & & & \\
C_{12} & C_{11} & C_{13} & & & \\
C_{13} & C_{13} & C_{33} & & & \\
& & & C_{44} & & \\
& & & & C_{44} & \\
& & & & & \left(C_{11}-C_{12}\right) / 2
\end{array}\right]\left\{\begin{array}{c}
e_{11} \\
e_{22} \\
e_{33} \\
2 e_{23} \\
2 e_{13} \\
2 e_{12}
\end{array}\right\}
$$

As indicated in Equation 12, for hexagonal symmetry there are in general five independent moduli. The elastic behavior can be decomposed into deviatoric (shear) and spherical (bulk) parts provided that the elastic moduli satisfy the constraint:

$$
C_{11}+C_{12}=C_{13}+C_{33}
$$

This constraint was imposed here, leaving four independent moduli. The bulk response is isotropic (no orientation dependence), as is apparent from its equation involving only first invariants of the stress and strain:

$$
\operatorname{tr}(\boldsymbol{\tau})=\frac{\kappa}{3} \operatorname{tr}\left(e^{e}\right)
$$

where the scalar bulk modulus, $\kappa$, is a linear combination of the $C_{11}, C_{12}$, and $C_{13}$ :

$$
\kappa=3\left(C_{11}+C_{12}+C_{13}\right)
$$

The single crystal elastic anisotropy is expressed entirely through a tensor relation between the deviatoric parts of the stress and strain.

The decomposition of Hooke's law into bulk and deviatoric parts has important implications relative to the harmonic expansions of the lattice strains and the subsequent determination of the elastic moduli using the expansions. Namely, it is the bulk modulus that is sensitive to the variability in the first mode coefficients (recall that the first mode of the harmonic expansion is simply a constant). However, with Hooke's law written in the form of Equation 12 that combines the bulk and deviatoric responses, uncertainty in the bulk response may also influence determination of moduli that associated with the deviatoric response. This is because in Equation 12 the three single crystal moduli that define the bulk response also influence the deviatoric response. This is in contrast to Equation 14 that isolates the bulk response using the single parameter, $\kappa$. 
The consequence of uncertainty in the first mode of the expansion, then, is that using the SPF data reported here, it was not possible to reliably estimate of the bulk modulus. However, because the high level of uncertainty was limited to the first mode, it was possible to estimate moduli associated with the deviatoric (anisotropic) response. This was accomplished by constraining the values of $C$ in the optimization procedure according to Equation 15 to maintain a fixed value of the bulk modulus. The value was one chosen from the literature for titanium [27]. At first this appears to be a limitation of using this approach to determine the moduli. However, further consideration points to a definite strength of the harmonic expansions as an avenue to treat data. Without isolating the variability to the first mode, it was not possible to assign the uncertainty to the bulk response alone. Thus, we were able to separate the deviatoric response and evaluate moduli associated with that part of the full behavior.

\subsection{Optimized moduli}

The total error, $R_{w}$, was minimized between the experimental spherical harmonic coefficients (taken as the average from the 6 diffraction volumes) and the simulated spherical harmonic coefficients for the 15 SPF conditions (5 load points each for 3 crystal family planes) by adjusting the elastic shear moduli. For each SPF condition, the error was taken as the root sum of squares of the difference between the experiment and simulation. The total error, $R_{w}$, is the sum of all the errors across all SPF conditions. 42 simulations were run to optimise the elastic moduli, with the initial $R_{w}$ of $30.6 \times 10^{-4}$ reduced to a final $R_{w}$ of $12.9 \times 10^{-4}$. The resulting strain pole figures for the optimized moduli are shown in Figure 8. These SPFs are virtually indistinguishable from the measured ones shown in Figure 6.

The expansions were dominated by contributions from the fifth and sixth modes, and so we focus on comparisons of the harmonic expansion coefficients for these two modes. Figure 9 shows a good match between experimental and simulated responses for modes five and six. Over the entire elastic domain, the coefficients for the simulated responses using the optimized moduli tract the experimental coefficients closely. In contrast, as can also be seen in Figure 9, there are clear differences in the trends for modes five and six for the responses computed with the moduli given for titanium in Kelly and Groves [27]. In particular, the magnitudes of contributions for both modes are substantially greater in the simulated behavior than is observed in experiment for the $\left\{\begin{array}{llll}0 & 0 & 0 & 2\end{array}\right\}$ crystal plane family.

The result of the optimization procedure is a set of moduli, $C_{11}, C_{12}, C_{13}$ 


\begin{tabular}{llllll} 
& \multicolumn{5}{c}{ Macroscopic Stress ( MPa ) } \\
\hline hkil & 270 & 383 & \\
\hline
\end{tabular}

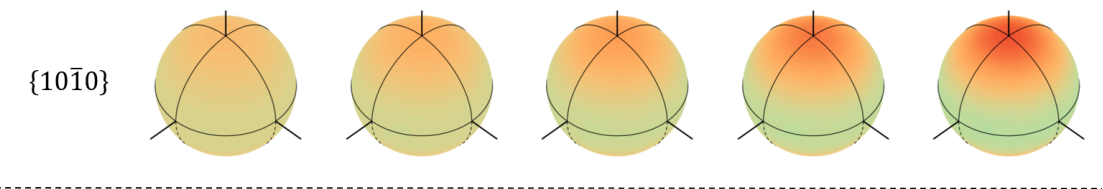

$\{10 \overline{1} 1\}$

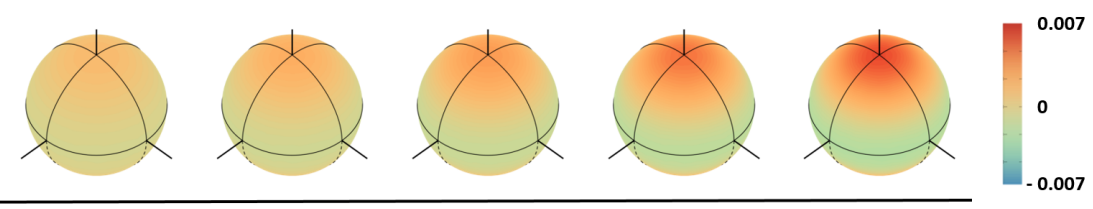

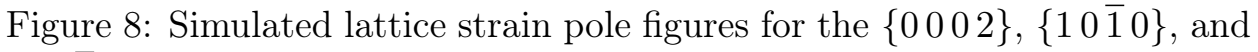
$\{10 \overline{1} 1\}$ crystal plane families at five macroscopic true stress values in the elastic region of the macroscopic true stress-strain response.

and $C_{44}$, that best describe the elastic responses quantified by the lattice strain distributions measured in the HEXD experiments. This is a very demanding test given the extensive number of independent measurements generated in these experiments. The optimized moduli are given in Table 1. As can be seen from comparisons of values in the table, the optimized values overall are in general agreement with the literature values. However, because the bulk moduli for the two sets are the same, the differences in the two sets of values lie in the deviatoric responses. Here, the difference between the two sets is about $10 \%$, which is directly evident in the $C_{44}$ values but is present in the relative values of the other moduli, as well.

\begin{tabular}{||c|c|c|c|c|c|c||}
\hline \hline Case & $C_{11}$ & $C_{12}$ & $C_{13}$ & $C_{44}$ & $\frac{E_{\max }}{E_{\min }}$ & $\frac{G_{\max }}{G_{\min }}$ \\
\hline \hline Kelly/Groves & 161 & 91 & 70 & 47 & 1.360 & 1.367 \\
\hline Optimized & 169 & 89 & 62 & 43 & 1.494 & 1.399 \\
\hline \hline
\end{tabular}

Table 1: Optimized and reference single crystal elastic moduli (GPa) 

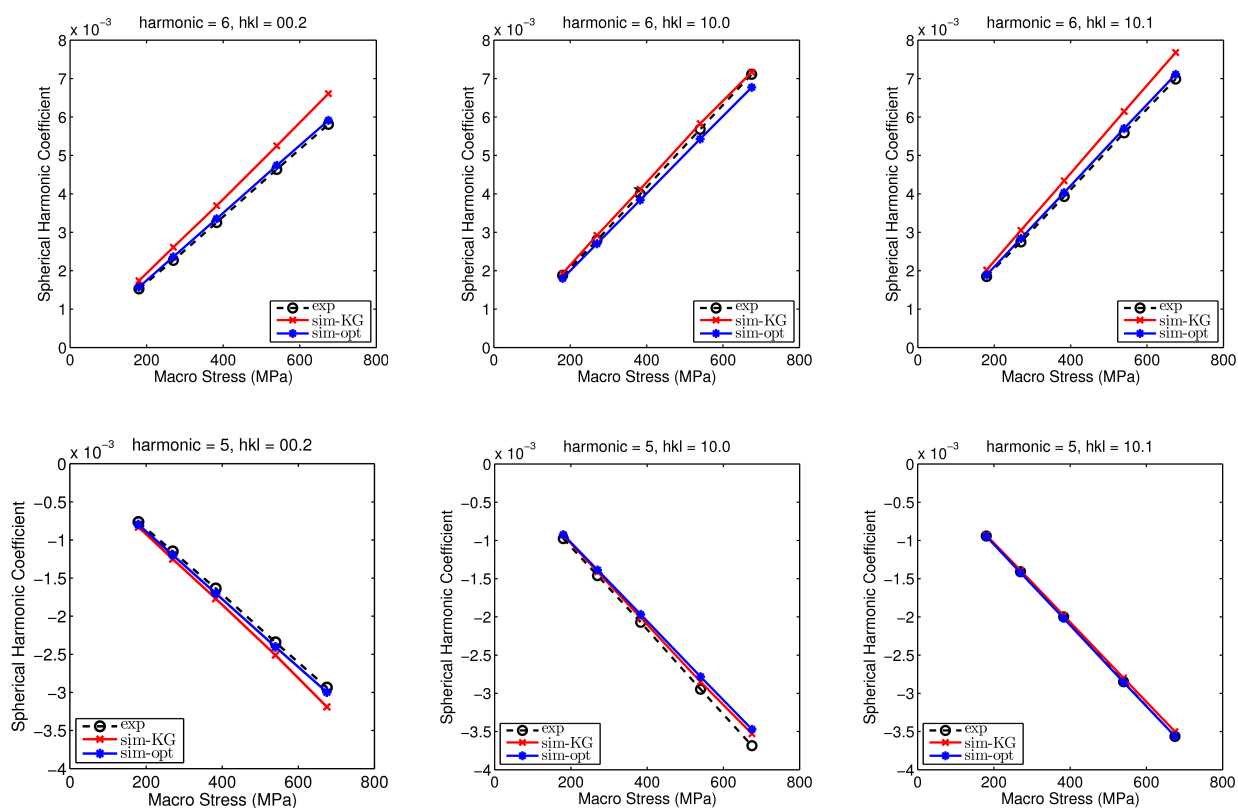

Figure 9: Coefficients of discrete spherical harmonics 5 and 6 for the $\left\{\begin{array}{lll}0 & 0 & 0\end{array}\right\}$, $\{10 \overline{1} 0\}$, and $\{10 \overline{1} 1\}$ crystal plane families plotted against macroscopic true stress calculated from experiments and simulations using the original Kelly and Groves moduli and the optimized moduli.

\section{Discussion}

Although the differences between the base (Kelly and Groves) and optimized moduli given in Table 1 are relatively small, these differences result in subtle but significant changes to the elastic response of the HCP unit cell. To quantify these subtle changes to the elastic response, we can define a directional elastic modulus and shear modulus that capture the elastic response of the HCP unit cell. The directional modulus is defined as $1 / S_{33}$, where $S_{33}$ is the directional component of the rotated compliance matrix. Likewise, a shear modulus for the HCP unit cell can be defined as $1 / S_{44}$, where $S_{44}$ is the shear component of the rotated compliance matrix. The direction and shear moduli can then be normalized and plotted over the fundamental region of Rodrigues space, as shown in Figure 10 for the cases of the base (Kelly and Groves) elastic constants, the optimized elastic constants and those for zirconium and magnesium [1].

From the plots shown in Figure 10, a number of interesting observations 


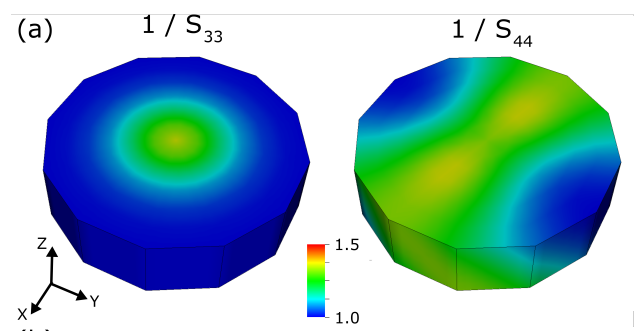

(b)

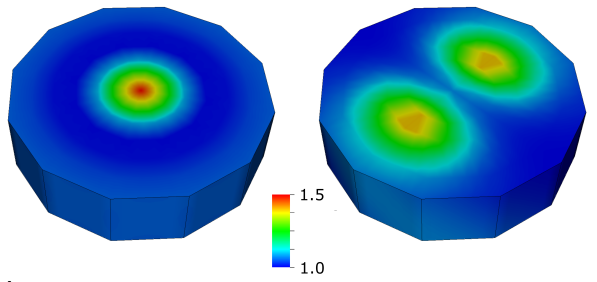

(c)

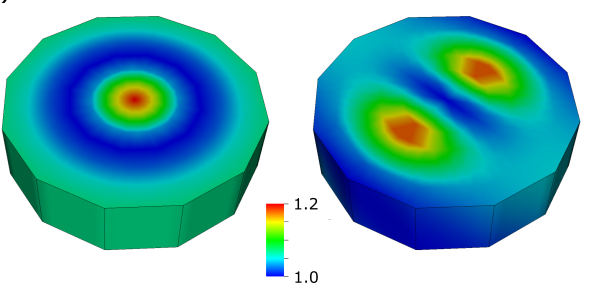

(d)

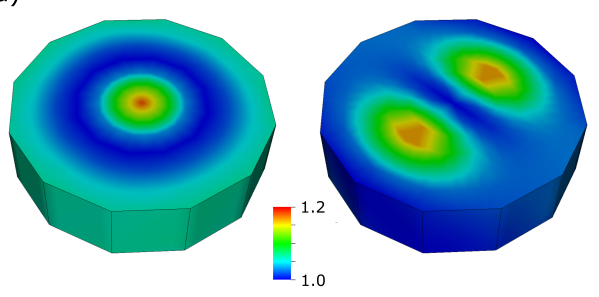

Figure 10: Plots showing the normalized directional modulus $\left(1 / S_{33}\right)$ and shear modulus $\left(1 / S_{44}\right)$ over the hexagonal fundamental region of Rodrigues space for (a) titanium (Kelly and Groves), (b) titanium (optimized), (c) zirconium and (d) magnesium.

can be made about the elastic response given by the base (Kelly and Groves) moduli compared with the optimized moduli, particularly in relation to the equivalent plots for magnesium and zirconium. The directional modulus for the base (Kelly and Groves) moduli is seen to monotonically decrease as it reaches the edges of the fundamental region. In contrast, although difficult to see due to the color scaling, the directional modulus for the optimized moduli has a minimum before increasing slightly as it reaches the edge of 
the fundamental region. Interestingly, this response for the directional modulus for the optimized moduli shows the same trend as for magnesium and zirconium [28]. Comparing the shear moduli plots for the base (Kelly and Groves) and optimized moduli, we can again see that there are differences between the responses. Comparing with the equivalent plots for magnesium and zirconium, it is once again interesting to note that the optimized moduli show the same trend in shear response.

It is worth highlighting that this is the first time this analysis has been conducted and, although efforts have been made to quantify errors, further experiments are required to validate the technique and assess it's applicability across different materials systems. Further, the BCC $\beta$ phase of Ti-6Al$4 \mathrm{~V}$ was neglected in the optimization. Although the $\beta$ phase accounts for only $7-8 \%$ of the material, it does contribute to the overall elastic response of Ti-6Al-4V. However, for the determination of the moduli for the $\alpha$ phase, it is uncertain how much the inclusion of the $\beta$ would change the response of the HCP $\alpha$ phase, particularly in terms of the spherical harmonics of the lattice SPFs. In future studies, the authors intend to extend this analysis technique to include the $\beta$ phase, with the aim of determining the extent of the $\beta$ phase's role in the elastic response of Ti-6Al- $4 \mathrm{~V}$ and to find optimal elastic moduli for the $\beta$ phase.

\section{Conclusions}

In this paper we have presented a new methodology for extracting single crystal elastic moduli from mechanical tests on polycrystalline samples and coordinated simulations of virtual polycrystals. The experiments use high energy x-ray, powder diffraction measurements during in situ mechanical loading to give extensive sets of lattice strain distributions. The simulations of virtual polycrystals mimic the mechanical loading of the experiments, producing similar lattice strain distributions.

The new method provides a framework to evaluate moduli by minimizing the difference between the measured and predicted responses. Central to the method is the use of a discrete spherical harmonic expansion of SPFs that permits comparison of the entire lattice strain distributions on the basis of the expansion coefficients. Further, the use of the spherical harmonic expansion allows decomposition of the lattice strain response to isolate the bulk and shear moduli.

The method has been successfully applied to the microstructurally complex engineering alloy, Ti-6Al-4V, with optimized single crystal moduli ex- 
tracted for the $\mathrm{HCP} \alpha$ phase. It is envisioned that this approach can be applied to a wide range of engineering materials where determining single crystal elastic moduli using traditional techniques is generally not possible.

\section{Acknowledgements}

This work was supported by the US Office of Naval Research under award N00014-12-1-0399. This work is based upon research conducted at the Cornell High Energy Synchrotron Source (CHESS) which is supported by the

National Science Foundation and the National Institutes of Health/National Institute of General Medical Sciences under NSF award DMR-1332208.

\section{References}

[1] R. F. S. Hearmon. The elastic constants of anisotropic materials. Rev. Mod. Phys., 18:409-440, Jul 1946.

[2] P.W. Bridgman. Some properties of single metal crystals. National Academy of Sciences - Proceedings, 10(10):411 - 415, 1924.

[3] P.W. Bridgman. Certain physical properties of single crystals of tungsten, antimony, bismuth, tellurium, cadmium, zinc, and tin. American Academy of Arts and Sciences - Proceedings, 60(6):305 - 383, 1925.

[4] W Voigt. A determination of the elastic constants for beta-quartz lehrbuch de kristallphysik. Terubner, Leipzig, 1928.

[5] Ludwig Bergmann, Henry Hatfield, et al. Ultrasonics and their scientific and technical applications. G. Bell and Sons Ltd., 1938.

[6] H.B. Huntington. Ultrasonic measurements on single crystals. Physical Review, 72(4):321 - 331, 1947.

[7] William C Schneider and Charles J Burton. Determination of the elastic constants of solids by ultrasonic methods. Journal of Applied Physics, 20(1):48-58, 1949.

[8] H.J. McSkimin. Wave propagation and measurement of elastic properties of liquids and solids. Acoustical Society of America - Journal, 28(6):1228 - 1232, 1956. 
[9] E. S. Fisher and C. J. Renken. Single-crystal elastic moduli and the hcp $\rightarrow$ bcc transformation in ti, zr, and hf. Phys. Rev., 135:A482-A494, Jul 1964 .

[10] E Griineisen and E Goens. Researches on metal crystals. i. elastic constants of zinc and cadmium. Zeitschrift fir Physik, 26:235, 1924.

[11] E Goens and J Weerts. Elastic constants of single crystals of copper, gold, and lead. Physik. Zeits, 37:321-420, 1936.

[12] J Y Kim, V Yakovlev, and S I Rokhlin. Line-focus acoustic microscopy of Ti-6242 alpha/beta single colony: determination of elastic constants. In Donald O Thompson, Dale E Chimenti, Linda Poore, Connie Nessa, and Sarah Kallsen, editors, Line-focus acoustic microscopy of Ti-6242 alpha/beta single colony: determination of elastic constants, pages 11181125. AIP, 2002.

[13] H Poulsen. Three-Dimensional X-Ray Diffraction Microscopy. Springer, Heidelberg, U.K., 2004.

[14] C Efstathiou, D. E. Boyce, J S Park, U Lienert, P R Dawson, and M P Miller. A method for measuring single crystal elastic moduli using high energy x-ray diffraction and a crystal-based finite element model. Acta Metallurgica et Materialia, 58(17):5806-5819, 2010.

[15] F. Bollenrath, V. Hauk, and E.H. Mueller. Zur berechnung der vielkristallinen elastizitaetskonstanten aus den werten der einkristalle. Zeitschrift fuer Metallkunde, 58(1):76 - 82, 1967.

[16] V. Hauk and H. Kockelmann. Evaluation of single crystal elastic constants from mechanical and x-ray elastic constants of the polycrystal. Z. Met. kd. (West Germany), 70(3):500 - 2, 1979.

[17] Anil K. Singh, Ho-kwang Mao, Jinfu Shu, and Russell J. Hemley. Estimation of single-crystal elastic moduli from polycrystalline x-ray diffraction at high pressure: Application to feo and iron. Phys. Rev. Lett., 80:2157-2160, Mar 1998.

[18] Thomas Gnäupel-Herold, Paul C. Brand, and Henry J. Prask. Calculation of Single-Crystal Elastic Constants for Cubic Crystal Symmetry from Powder Diffraction Data. Journal of Applied Crystallography, 31(6):929-935, Dec 1998. 
[19] C. J. Howard and E. H. Kisi. Measurement of single-crystal elastic constants by neutron diffraction from polycrystals. Journal of Applied Crystallography, 32(4):624-633, Aug 1999.

[20] M P Miller, J. V. Bernier, J S Park, and A Kazimirov. Experimental measurement of lattice strain pole figures using synchrotron $\mathrm{x}$ rays. Review of Scientific Instruments, 76(11):113903, 2005.

[21] M P Miller, J S Park, P R Dawson, and T S Han. Measuring and modeling distributions of stress state in deforming polycrystals. Acta Metallurgica et Materialia, 56(15):3927-3939, 2008.

[22] H.-J. Bunge. Texture Analysis in Materials Science: Mathematical Methods. Elsevier, 2013.

[23] A Kumar and P.R Dawson. Computational modeling of f.c.c. deformation textures over rodrigues' space. Acta Materialia, 48(10):2719-2736, 62000 .

[24] G. Ischia, H.-R. Wenk, L. Lutterotti, and F. Berberich. Quantitative rietveld texture analysis of zirconium from single synchrotron diffraction images. Journal of Applied Crystallography, 38:377, 2005.

[25] Jay C Schuren, Su L Wong, Paul R Dawson, and Matthew P Miller. Integrating experiments and simulations to estimate uncertainty in lattice strain measurements. The Journal of Strain Analysis for Engineering Design, page 0309324713492325, 2013.

[26] P. Dawson and D. Boyce. FEpX - Finite Element Polycrystals: Theory, finite element formulation, numerical implementation and illustrative examples. arXiv:1504.03296 [cond-mat.mtrl-sci], 2015.

[27] A. Kelly and G. W. Groves. Crystallography and Crystal Defects. John Wiley and Sons, 1970.

[28] Desmond Tromans. Elastic anisotropy of HCP metal crystals and polycrystals. International Journal of Recent Research and Applied Studies, 6(4):462-483, 2011.

[29] F. C. Frank. Orientation mapping. Metallurgical Transactions A, 19(3):403-408, 31988.

[30] R Becker and S Panchanadeeswaran. Crystal rotations represented as rodrigues vectors. Textures and Microstructures, 10(3):167-194, 1989. 
[31] A. Heinz and P. Neumann. Representation of orientation and disorientation data for cubic, hexagonal, tetragonal and orthorhombic crystals. Acta Crystallographica Section A Foundations of Crystallography, 47(6):780-789, 111991.

[32] A Morawiec. Distributions of misorientation angles and misorientation axes for crystallites with different symmetries. Acta Crystallographica Section A, 53(3):273-285, 1997.

[33] A. Kumar and P. R. Dawson. Modeling crystallographic texture evolution with finite elements over neo-Eulerian orientation spaces. Computer Methods in Applied Mechanics and Engineering, 153:259-302, 1998.

[34] K. Atkinson and W. Han. Spherical harmonics and approximations on the unit sphere: An introduction, 2012. Lecture Notes in Mathematics, Volume 2044, ISBN 978-3-642-25982-1 (Print) 978-3-642-25983-8 (Online).

\section{Appendices}

\section{A Crystal orientations and lattice strains}

The orientation of the crystallographic lattice is quantified using an angleaxis parameterization to relate a set of basis vectors attached to the lattice to a common fixed reference frame. Rodrigues vectors, $\boldsymbol{r}$, are angle-axis representations of orientations defined by:

$$
\boldsymbol{r}=\tan \left(\frac{\phi}{2}\right) \boldsymbol{n}
$$

in which $\boldsymbol{n}$ is the rotation axis and $\phi$ is the rotation angle [29]. Using crystal symmetries to define bounding planes, a fundamental region in orientation space, $\Omega_{\mathrm{fr}}$, may be determined with the property that all points on the interior of the region represent unique orientations. Points on the boundary are duplicated by other boundary points according to crystal symmetries [3032]. The fundamental region for hexagonal crystal symmetry is shown in Figure 11. Continuous distributions over the fundamental region are constructed with linear piecewise polynomials (e.g. lower-order finite element interpolation functions) [33].

Using a Rodrigues parameterization of orientations, crystallographic fibers are straight lines in orientation space. Several examples from the hexagonal 


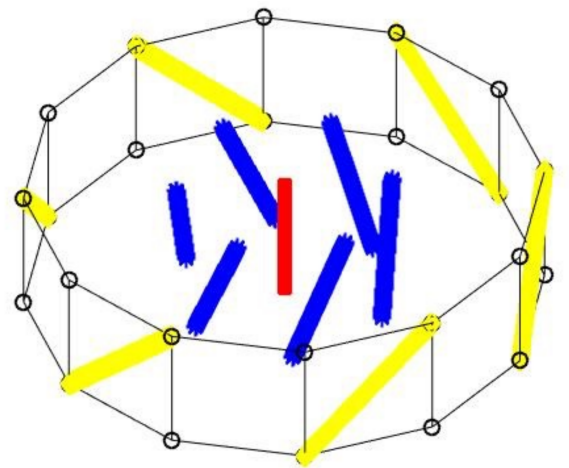

Figure 11: Fundamental region of Rodrigues space for hexagonal symmetry

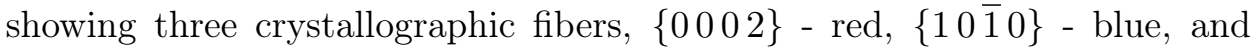
$\{10 \overline{1} 1\}$ - yellow, with crystal plane normals parallel to the sample $x_{3}$ direction.

case, namely $\left\{\begin{array}{lll}0 & 0 & 2\end{array}\right\},\{10 \overline{1} 0\}$, and $\{10 \overline{1} 1\}$, with crystal plane normals parallel to the sample $x_{3}$ direction, are shown in Figure 11. Utilizing the Rodrigues parameterization of orientations and the finite element representation of the fundamental region significantly simplifies calculations over orientation space, such as the integration of quantities along crystallographic fibers.

\section{B Discrete Spherical Harmonics}

One approach in comparing two distributions is to examine the coefficients associated with series expansions of the distributions. Similarity of matching coefficients implies that the two distributions both possess a variation over the domain that is captured by the corresponding term in the expansion. In this appendix we develop the discrete spherical harmonic expansion functions that are used in Section 2 to compare simulated and measured strain distributions via the coefficients in their series representations.

Solid harmonics are homogeneous polynomials with zero Laplacian [34]. This means that, over the solid domain $\Omega$, a solid harmonic, $u$, that is homogeneous of degree $m$ must satisfy:

$$
\begin{aligned}
\nabla^{2} u & =0 \\
u(\lambda x) & =\lambda^{m} u(x)
\end{aligned}
$$


Spherical harmonics are solid harmonics that are constrained to lie on the surface of a unit sphere, designated as $\Gamma$. Note that the domain of the solid may be defined in any number of dimensions, $R^{n+1}$, with $n \geq 1$. The surface of the sphere exists in a space, $S^{n}$, that is one lower in dimension. For representation of the strain distributions as a function of the sample direction $s, n=2$.

Because of the homogeneity of the solid harmonics, their normal derivatives are proportional to their values. This leads to the requirement that, on the sphere surface, $\Gamma$, the spherical harmonic functions satisfy:

$$
-\nabla^{2} u=\lambda u
$$

From this requirement we can identify the spherical harmonics as the eigenfunctions of Equation 19. Using the spherical harmonic functions as bases of the series expansion allows us to write $u$ as:

$$
u=\sum_{k=1}^{n^{w}} w^{k} H^{k}(s) \quad \text { on } \Gamma
$$

where $w^{k}$ are the weights and the eigenvectors, $H^{k}(s)$, lie on the sphere and are smooth. The expansion is limited to $n_{w}$ terms using the lower-mode eigenvectors. As a consequence of being the eigenvectors of Equation 19, the functions admit the following orthogonality:

$$
\left.\int_{\Gamma} H^{i}(\boldsymbol{s}) H^{j} \boldsymbol{s}\right) \mathrm{d} s= \begin{cases}1 & i=j \\ 0 & i \neq j\end{cases}
$$

Equation 19 may be solved for the spherical harmonics using finite elements. To do this, a weighted residual is constructed from Equation 19 using the weights, $\Psi(s)$, as:

$$
\int_{\Gamma} \Psi(s)\left(\nabla^{2} u+\lambda u\right) \mathrm{d} s=0
$$

and subsequently is modified to obtain its weak form. The domain, $\Omega_{s}$, is discretized with elements to create a finite element mesh and $H^{k}(\boldsymbol{s})$ is represented over the mesh using piecewise polynomial approximation, $\hat{H}^{k}(s)$, with $C^{0}$ continuity:

$$
\hat{H}^{k}(s)=\left[N^{s}(s)\right]\left\{\hat{H}^{k}\right\}
$$


Here, $\left[N^{s}(s)\right]$ are interpolation functions over the element domains and $\left\{\hat{H}^{k}\right\}$ are the associated nodal point values.

Applying a Galerkin approach, the weights are defined using the same interpolation functions:

$$
\hat{\Psi}(s)=\left[N^{s}(s)\right]\left\{\hat{\psi}^{k}\right\}
$$

These discrete representations of $H^{k}(s)$ and $\Psi(s)$ are substituted into the weak form of the residual to obtain:

$$
\left[\left[K_{H}\right]-\lambda\left[M_{H}\right]\right]\left\{\hat{H}^{k}\right\}=\{0\}
$$

where $\left[K_{H}\right]$ and $\left[M_{H}\right]$ are the assembled elemental matrices:

$$
\left[K_{H}\right]=\sum_{n_{e}}\left[k_{H}\right] \quad \text { and } \quad\left[\mathrm{M}_{\mathrm{H}}\right]=\sum_{\mathrm{n}_{\mathrm{e}}}\left[\mathrm{m}_{\mathrm{H}}\right]
$$

The elemental matrices in turn are:

$$
\left[k_{H}\right]=\int_{\Gamma^{e}}[B]^{\mathrm{T}}[B] \mathrm{d} s
$$

and

$$
\left[m_{H}\right]=\int_{\Gamma^{e}}[N]^{\mathrm{T}}[N] \mathrm{d} s
$$

where $[B]$ are the gradients of $\left[N^{s}(s)\right]$ on the sphere.

Extracting the eigenvectors from Equation 25 provides a set of discrete spherical harmonics, $\hat{H}^{k}(s)$, which are tied explicitly to the finite element mesh used for Equation 23. The number of modes corresponds to the number of nodal points in the mesh. However, only a relatively small number of modes are needed to capture the dominant trends of a distribution. Here we employed the first 25 modes, as displayed in Figure 12.

\section{Experimental error analysis}

Since SPF measurements were made for six independent volumes in the gage section of the specimen at each load step, a statistical analysis can be conducted to determine the measurement error in the lattice strain at every nodal point. Assuming a normal distribution, the standard error at each nodal point was calculated with a confidence interval of $95 \%$. The results from this analysis were then combined to define a continuous standard error function over the unit sphere. The standard error can then be plotted as an 


\section{1 - Uniform Sphere}

2

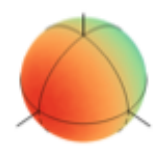

6

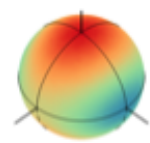

10

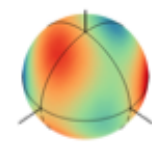

14

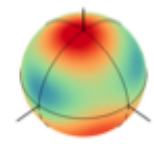

18

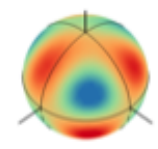

22

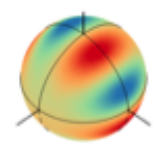

3

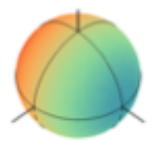

7

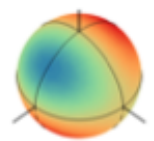

11

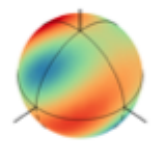

15

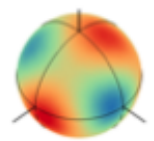

19

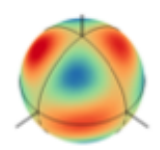

23

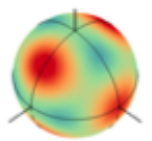

4

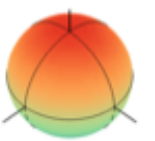

8

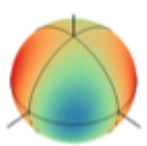

12

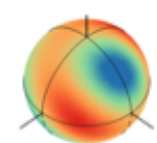

16

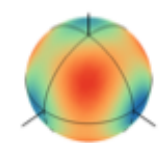

20

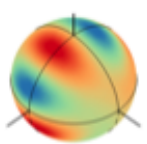

24

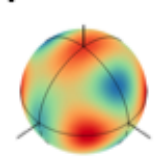

5

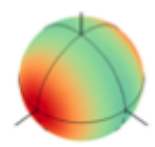

9

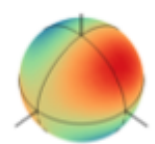

13

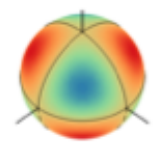

17

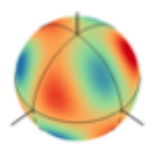

21

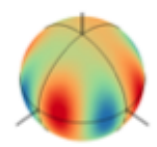

25

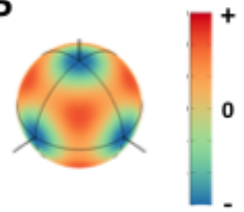

Figure 12: 25 discrete spherical harmonic modes used in this analysis. 
error pole figure (EPF) for each family of crystallographic planes and load step, as shown in Figure 13.

From Figure 13 it can qualitatively be seen that the distribution of standard error over the EPF is random but that the error distribution propagates through load steps. It can also be see that there are differences in the level of

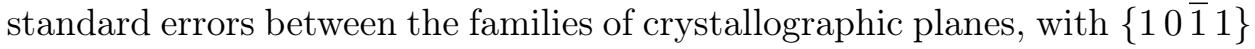
having the lowest peaks in standard error.

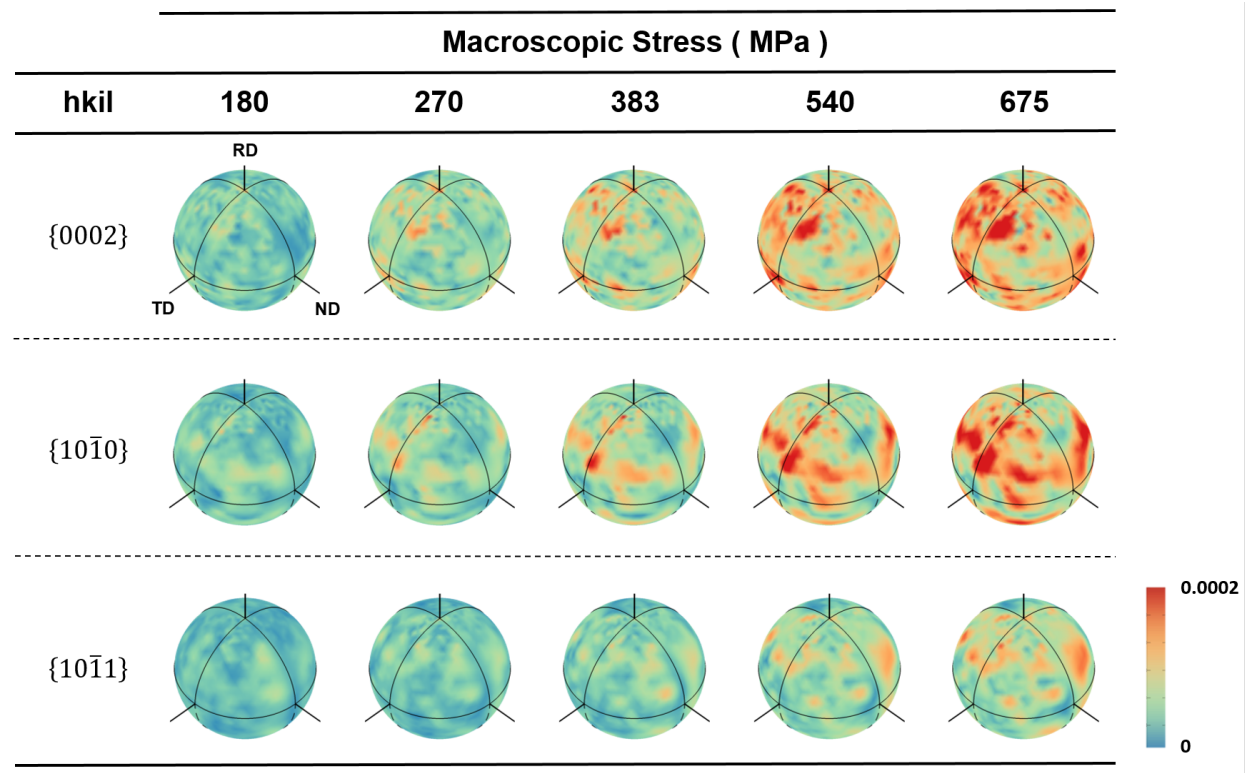

Figure 13: Standard error in the lattice strain pole figures for the $\left\{\begin{array}{lll}0 & 0 & 0\end{array}\right\}$, $\{10 \overline{1} 0\}$, and $\{10 \overline{1} 1\}$ crystal plane families at five macroscopic true stress values in the elastic region of the macroscopic true stress-strain response. 